EPJ Web of Conferences 45, 01018 (2013)

DOI: $10.1051 /$ epjconf/20134501018

(c) Owned by the authors, published by EDP Sciences, 2013

\title{
Permeability of mono- and bi-dispersed porous media
}

\author{
C. Byon ${ }^{1, a}$ and S.J. Kim ${ }^{2}$ \\ ${ }^{1}$ Yeungnam University, School of Mechanical Engineering, 712-749 Gyeongsan, Republic of Korea \\ ${ }^{2}$ KAIST, Mechanical Engineering Department, 305-701 Daejeon, Republic of Korea
}

\begin{abstract}
In this study, the permeability of mono- and bi-dispersed porous media is considered. The effects of the particle size distribution and the packing structure of particles on the permeability are investigated experimentally and analytically. Both experimental and analytic results suggest that the particle size distribution is close to the log-normal distribution, and the permeability of the mono-dispersed porous media quasi-linearly decreases as the range of the particle size distribution increases. On the other hand, the effect of packing structure of particles on the permeability is shown to be negligible. The permeability of the bidispersed porous media quasi-linearly decreases as the range of cluster size increases, and nearly independent of the particle size distribution. The present model is valid over the range of parameters typically found in heat transfer applications.
\end{abstract}

\section{Introduction}

Porous media with sintered microsized particles, have received considerable interests for heat transfer applications due to its ability to provide high capillary pumping pressure and high effective thermal conductivity $[1,2]$. The heat pipe is a well-known heat transfer device which is based on the capillary pumping of the porous media [3]. A key parameter that determines the capillary pumping ability of the porous media is the permeability [4], which relates the velocity of the flow and the applied pressure drop [5]. In order to predict the permeability of the porous media, the Blake-Kozeny's equation (equation 1) has been widely used [6].

$$
K=\frac{1}{150} \frac{\varepsilon^{3} d^{2}}{(1-\varepsilon)^{2}}
$$

where $K$ is permeability, $\varepsilon$ is porosity, and $d$ is the particle size. As shown in equation 1, the Blake-Kozeny's equation assumes that the particle diameter is constant. However, the microsized particles that constitute the porous media have a significant size distribution since they are generally produced by high pressure atomization and subsequent sieving process. Therefore, the BlakeKozeny's equation may result in a significant error when the range of the particle size distribution is not negligible compared with its mean value [8-10].

In addition, the packing structure of particles in sintered porous media is different from that of the packed bed and it is highly dependent on the sintering process. For instance, in the sintered porous media, the particles are not connected at a point contact but have bonded areas. To the authors' knowledge, no investigation has been performed to address the aforementioned concerns. In order to accurately predict the permeability of the sintered porous media, a model which takes into account the effects of particle size distribution and the packing structure should be developed.

This study aims to investigate the permeability of the sintered porous media with taking into account the particle size distribution and the packing structure. In this study, two distinct types of porous media are considered: The mono-dispersed porous media and the bi-dispersed porous media. The mono-dispersed porous media are conventional types of porous media, which consist of particles. The prefix 'mono' indicates that the microstructure has one characteristic length represented by the particle size. On the other hand, the bi-dispersed porous media have two distinct characteristic lengths and generally consist of agglomerated clusters of small metal particles. The two characteristic lengths indicate the particle size and the cluster size, accordingly. The bidispersed porous media are known to provide attractive properties such as high permeability and efficient vapor escape. Typical configurations of mono- and bi-dispersed porous media are illustrated in figure 1 . In this study, the effects of the particle size distributions and the packing structure of mono- and bi-dispersed porous media are investigated experimentally and analytically. The porous media with various particle size distributions are prepared by sieving the raw particles into various fractions. The size distribution of the particles that constitute each porous medium is quantitatively measured using a particle size analyzer and the distribution parameters are extracted from the result. Based on the estimated particle

\footnotetext{
a c.byon@imperial.ac.uk
} 
size distribution, an analytic model for predicting the permeability of porous media is developed. In addition, the effect of the packing structure of the particles on the permeability of the porous media is investigated.

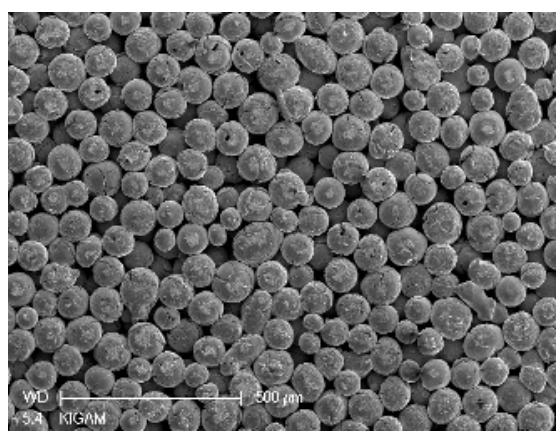

(a) Mono-dispersed

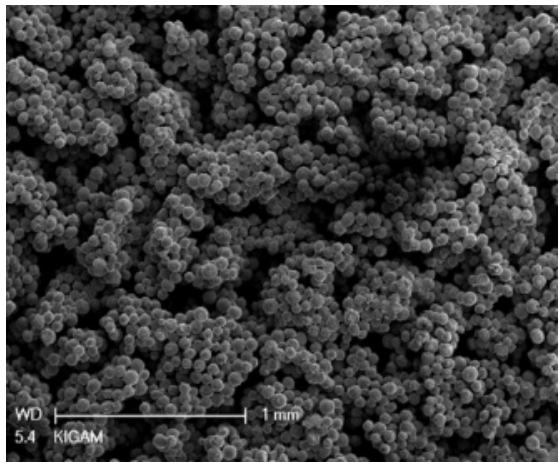

(b) Bi-dispersed

Fig. 1 SEM images of fabricated porous media

\section{Experiment}

To fabricate the mono- and bi-dispersed porous media, spherical copper particles (ACupowder, Inc.) are sintered into a porous medium in a high temperature furnace with a reducing atmosphere of nitrogen and hydrogen. The mono-dispersed porous media is fabricated by directly sintering the as-received particles. To fabricate the bidispersed porous media, the mono-dispersed porous medium is grinded into clusters, sieved into specific sizes, and sintered again. In this study, cylindrical samples are fabricated with $11 \mathrm{~mm}$ diameter and $30 \mathrm{~mm}$ length. Figure 1 shows SEM images of the fabricated monodispersed and bi-dispersed porous media. The particle size and a measure of the size distribution range normalized by its mean value are summarized in Table 1 , where $D_{\mathrm{p}}$ is mean particle diameter, and $\Delta D_{\mathrm{p}}{ }^{*}$ the standard deviation of particle size distribution normalized by the mean particle diameter. Accordingly, $D_{\mathrm{c}}$ and $\Delta D_{\mathrm{c}}{ }^{*}$ (Table 2) are the mean cluster size and the standard deviation normalized by mean cluster size, respectively. In this study, 9 mono-dispersed porous media and $10 \mathrm{bi}$ dispersed porous media $\left(D_{\mathrm{p}}=117 \mu \mathrm{m}\right.$ and $\left.D_{\mathrm{c}}=675 \mu \mathrm{m}\right)$ are tested. The size distributions of the particles and clusters are determined using the particle size analyzer (Beckman Coulter, LS230) which is based on the laser diffraction principle.
The experimental apparatus is illustrated in figure 2. It consists of a constant temperature water bath, a micro manometer, a mass flow meter, a PC, and stainless steel tubing. Water flow is maintained at a constant temperature using the water circulation bath (JeioTech, HTBC-2320AT). A mass flow meter (MFC, WinTEC) is used to measure the flow rate. Its accuracy and repeatability are $\pm 1 \%$ and $\pm 0.15 \%$, respectively. The stainless steel tube and the glass tube which is the shell of the test sample are carefully attached each other and sealed. The outer diameters of stainless steel tube and glass tube are both set to be $12.7 \mathrm{~mm}$ to eliminate the head pressure loss. To measure the pressure at the inlet and the outlet of the test sample, a micro-manometer (KIMO, MP112) is used. The measurement range and the accuracy of the micro-manometer is $200,000 \mathrm{~Pa}$ and $\pm 0.5 \%$, respectively. The velocity at the test sample can be readily evaluated from the mass flow rate and the cross sectional area of the test sample. From the measured velocity and the pressure difference between inlet and outlet, the permeability of the test sample can be obtained using the Darcy's equation:

$$
u=-\frac{K}{\mu} \frac{\Delta P}{\Delta x}
$$

where $K$ is permeability, $u$ is the area-averaged velocity of water, $\mu$ is dynamic viscosity, $\Delta P$ is pressure loss along the sample, and $\Delta x$ is the length of the sample.

Table 1. Size ranges of fabricated mono-dispersed porous media

\begin{tabular}{ccc}
\hline$\#$ & $D_{\mathrm{p}}(\mu \mathrm{m})$ & $\Delta D_{\mathrm{p}}{ }^{*}$ \\
\hline 1 & 90 & 0.178 \\
2 & 117 & 0.368 \\
3 & 153 & 0.307 \\
4 & 178 & 0.404 \\
5 & 180 & 0.111 \\
6 & 181 & 0.171 \\
7 & 225 & 0.333 \\
8 & 250 & 0.200 \\
9 & 350 & 0.429 \\
\hline
\end{tabular}

Table 2. Size ranges of fabricated bi-dispersed porous media $\left(D_{\mathrm{p}}=117 \mu \mathrm{m}, D_{\mathrm{c}}=650 \mu \mathrm{m}\right)$

\begin{tabular}{ccc}
\hline$\#$ & $\Delta D_{\mathrm{p}}{ }^{*}$ & $\Delta D_{\mathrm{c}}{ }^{*}$ \\
\hline 10 & 0.176 & 0.223 \\
11 & 0.176 & 0.259 \\
12 & 0.176 & 0.387 \\
13 & 0.254 & 0.223 \\
14 & 0.254 & 0.259 \\
15 & 0.254 & 0.387 \\
16 & 0.348 & 0.223 \\
17 & 0.348 & 0.259 \\
18 & 0.418 & 0.223 \\
19 & 0.418 & 0.259 \\
\hline
\end{tabular}




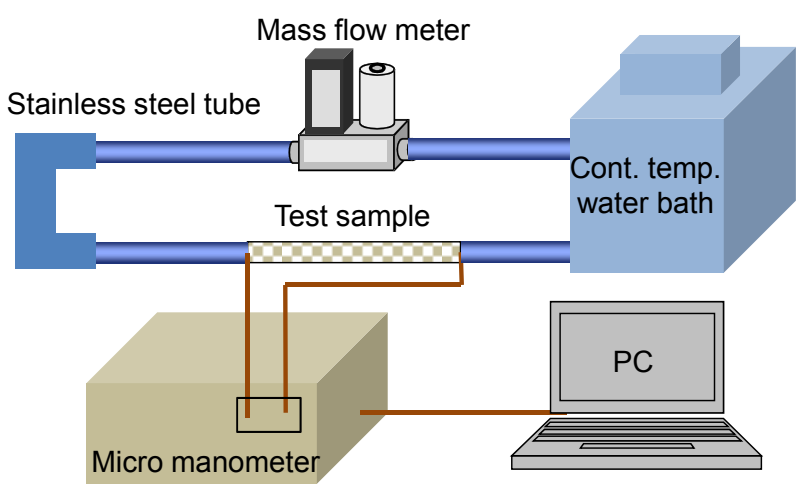

Fig. 2 Schematic diagram of the experimental apparatus deviation (MAD) of the best fit is determined to be less than $4 \%$. Therefore, the size distribution of sieved particles can be well-characterized by the log-normal distribution.

Table 3. Comparison between analytically and experimentally obtained distribution parameters

\begin{tabular}{|c|c|c|c|c|c|c|}
\hline \multirow{2}{*}{} & \multicolumn{3}{|c|}{ Analysis } & \multicolumn{3}{c|}{ Experiment } \\
\cline { 2 - 7 } & $D_{\text {mode }}$ & $D_{\text {mean }}$ & $\sigma_{\text {stan }}$ & $D_{\text {mode }}$ & $D_{\text {mean }}$ & $\sigma_{\text {stan }}$ \\
\hline 1 & 125.9 & 134.6 & 28.7 & 127.6 & 138.7 & 29.6 \\
\hline 2 & 162.3 & 166.8 & 22.6 & 163.9 & 169.1 & 22.0 \\
\hline 3 & 169.3 & 172.2 & 18.5 & 168.9 & 173.7 & 17.5 \\
\hline
\end{tabular}

\section{Analysis}

To predict the permeability of packed beds consisting of multi-sized particles, MacDonald et al. proposed an analytic expression based on the probability density theory [12]:

$$
K \cong \frac{1}{150} \frac{\varepsilon^{3}}{(1-\varepsilon)^{2}}\left(\frac{M_{2}}{M_{1}}\right)^{2}
$$

where $\varepsilon$ is the porosity, and $M_{i}$ is $i$ th moment of the particle size distribution defined as follows:

$$
M_{i}=\int_{0}^{\infty} D^{i} f(D) d D
$$

where $D$ indicates $D_{\mathrm{c}}$ or $D_{\mathrm{p}}$, and $f(D)$ represents the particle size distribution (fraction of particles whose diameters range between $D$ and $D+d D$ ). As the particle size distribution becomes narrower, the ratio $M_{2} / M_{1}$ in equation 3 becomes close to a mean particle diameter and thus, this equation approaches the Blake-Kozeny's equation.

The log-normal distribution is often used to approximate the particle size distribution of atomized particles, aerosols, aquatic particles and pulverized material [13].

$$
f(D)=\frac{A}{\sigma D \sqrt{2 \pi}} \exp \left[-\left\{\ln \left(\frac{D}{D_{m}}\right)\right\}^{2} / 2 \sigma^{2}\right]
$$

where $D_{\mathrm{m}}$ is median value, $\sigma$ is deviation constant and $A$ is curve area. $M_{1}$ and $M_{2}$ can be obtained by using equations 4-5. Mean, mode and standard deviation of the log-normal distribution can be obtained in analytic forms as shown in the following equations, respectively [14].

$$
\begin{gathered}
D_{\text {mean }}=D_{\mathrm{m}} \exp \left(\frac{\sigma^{2}}{2}\right) \\
D_{\text {mode }}=D_{\mathrm{m}} / \exp \left(\sigma^{2}\right) \\
\sigma_{\text {st an }}=D_{\mathrm{m}} \sqrt{\exp \left(\sigma^{2}\right)\left[\exp \left(\sigma^{2}\right)-1\right]}
\end{gathered}
$$

In Table 3, analytically (equation 6-8) and experimentally obtained distribution parameters are compared. As shown in this table, they match each other well. Figure 3 shows the experimentally obtained particle size distributions with best fits provided by equation 5 . The mean absolute

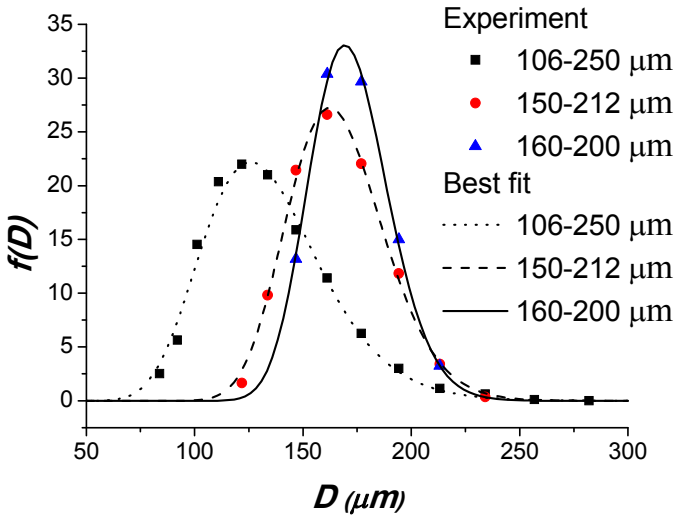

Fig. 3 Measured size distributions of the considered glass particles

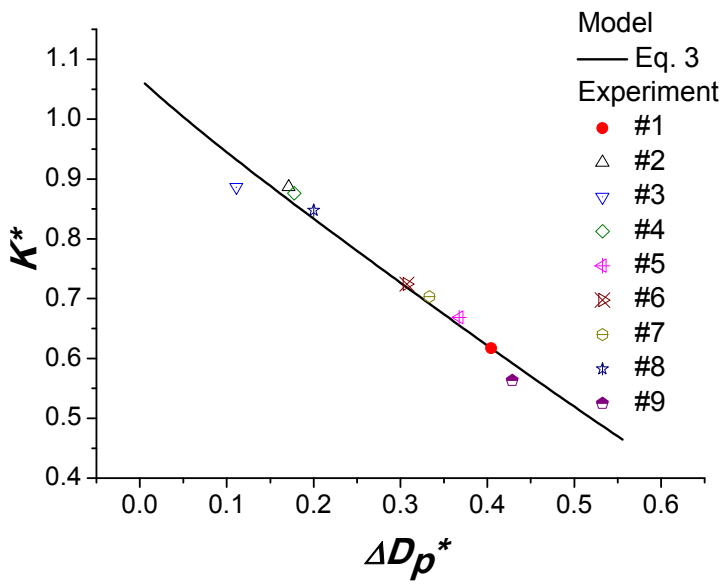

Fig. 4 Permeability versus particle size distribution (x-axis: nondimensional distribution range, $y$-axis: permeability normalized by the reference permeability, line: the present model result, dots: experimental result) 


\section{Numerical analysis}

Figure 4 shows a comparative plot of experimentally and analytically obtained permeabilities. In this figure, $K^{*}$ is a nondimensional measure of permeability, which is normalized by a reference value of permeability $K_{\text {ref. }}$ The $K_{\text {ref }}$ is estimated by the Blake-Kozeny's equation represented by equation 1 . As shown in this figure, the present analytic model predicts the experimental results well within the error of $5 \%$. The permeability quasilinearly decreases with increasing nondimensional particle size distribution range. When the nondimensional distribution range is 0.4 , the permeability decreases by $40 \%$ compared with the reference permeability. This is mainly due to the characteristics of the log-normal distribution that the median particle size decreases with increasing the distribution range. The present model is valid over the range of parameters considered in the experiment $(90 \mu \mathrm{m}$ $\left.<D_{\mathrm{p}}<380 \mu \mathrm{m}, 0.1<\Delta D_{\mathrm{p}}{ }^{*}<0.4\right)$, which is typically encountered in heat transfer applications. It should be noted that the present model cannot be applied to the particles made by mechanical grinding. When the particle size distribution deviates from the log-normal distribution, the results may be significantly altered.

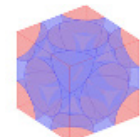

FCC

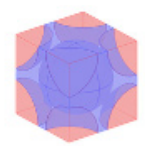

$\mathrm{BCC}$

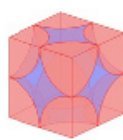

$\mathrm{SC}$ (a) The effect of the particle arrangement

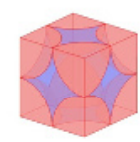

$R_{\mathrm{c}}{ }^{\circ}=0$

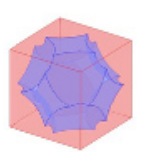

$R_{\mathrm{c}}{ }^{*}=0.3$

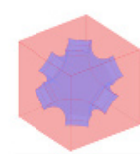

$R_{\mathrm{c}}^{*}=0.5$

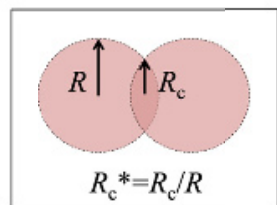

$R_{\mathrm{c}}{ }^{*}=R_{\mathrm{c}} / R$ (b) The effect of the bonded area

Fig. 5 Effect of particle arrangement and the bonded area (Unit cells are shown, FCC: Face-centered cubic, BCC: Bodycentered cubic, $\mathrm{SC}$ : Simple cubic, $R_{\mathrm{c}}$ : the ratio between radius of bonded area and particle radius)

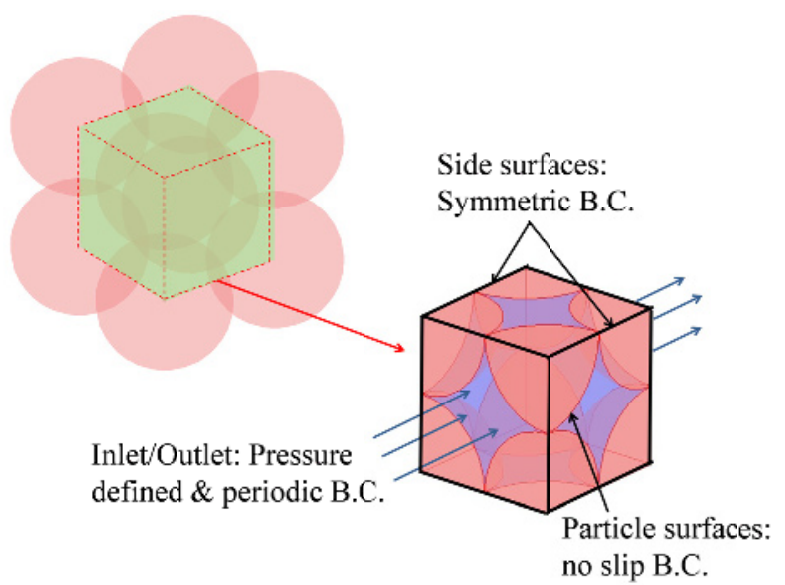

Fig. 6 Unit cell and boundary conditions used for numerical simulation

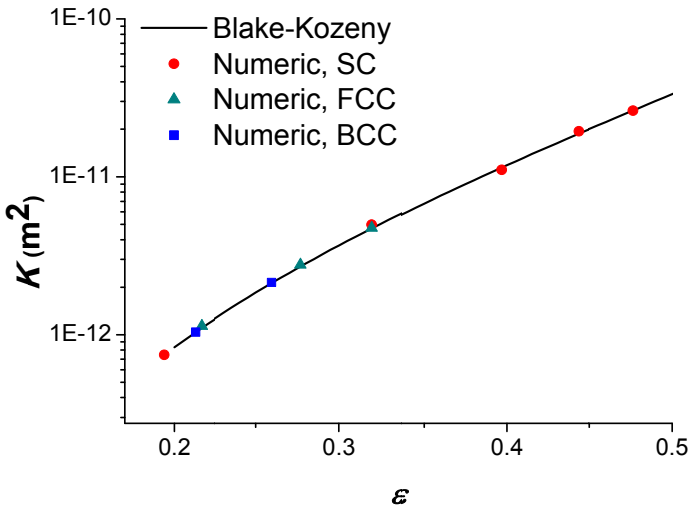

Fig. 7 Comparative plots of analytically and numerically obtained permeabilities versus porosity

Besides the effect of the size range, the effect of the packing structure of the sintered particles on the permeability of sintered porous medium is considered in this study. The sintered porous media can have different packing structures depending on the sintering condition. The packing structure of sintered porous medium can be represented by two geometric parameters: the particle arrangement and the particle-tio-particle bonded area [15]. When the particles are well shaken and compressed prior to the sintering, the particles get more closely arranged and the porosity decreases. As the sintering temperature or time increases, the bonded area becomes large, which in turn decreases the porosity. These parameters are wellcoupled each other, and may significantly affect the permeability predictions in terms of its scaling relation with the porosity. Therefore, the effects of the particle arrangement and the bonded area on the permeability should be investigated. Experimentally controlling these geometric parameters is difficult. Therefore, a numerical simulation for predicting the effect of those parameters is performed in this study. Figure 5 shows considered particle arrangements and the bonded areas. Three representative types of particle arrangement: the facedcentered cubic, body-centered cubic and simple cubic structures are considered. Even though the actual particle arrangement is expected to be different, the present numerical study can provide a qualitative understanding of how the particle arrangement affects the permeability. The effect of the bonded area are also considered by imposing various values of $R_{\mathrm{c}}{ }^{*}$, which is the ratio between the radius of the bonded area and the particle radius, as shown in figure 5(b). Figure 6 shows the unit cell and the boundary conditions for the numerical simulation. The no-slip boundary condition is applied to all solid surfaces, and the symmetry condition is applied to the side surfaces. A periodic pressure boundary condition was applied at the inlet and outlet. The steady incompressible Navier-Stokes equations (equation 9) are solved to predict the $3 \mathrm{D}$ velocity profiles and then compute the average velocity across the inlet. From the predicted average velocity, the permeability was then determined using Darcy's law (equation 2).

$$
\frac{\rho}{\varepsilon^{2}}(u \cdot \nabla u)=-\frac{\mu}{K_{\text {mono }}} u-\nabla p+\rho \vec{g}+\frac{\mu}{\varepsilon} \nabla^{2} u
$$


In the numerical study, the inlet pressure was adjusted to fix the Reynolds number at 0.1 which is a common value in micro-flow applications.

Figure 7 shows the predicted permeabilities and a curve representing the Blake-Kozeny's equation. As shown in this figure, the Blake-Kozeny's equation is shown to predict the permeabilities of sintered porous media well regardless of the particle arrangement and the bonded area. Therefore, the Blake-Kozeny's equation, which has been initially developed for the packed bed, can be also useful for the sintered porous media which have irregular particle arrangement together with nonzero particle-to-particle bonded area. However, the particle size distribution should be taken into account for an accurate prediction of the permeability.

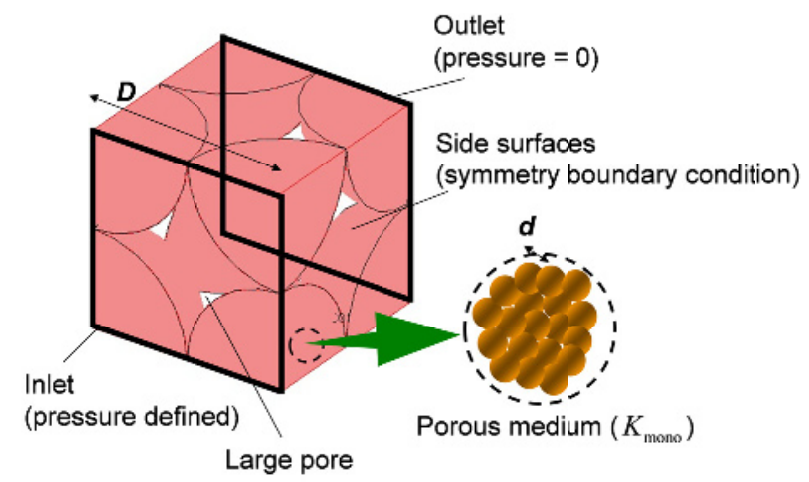

Fig. 8 Numerical simulation for the bi-dispersed medium

Based on the result that the permeability of the porous media is not meaningfully dependent on the packing structure, the investigation on the particle size distribution is extended for the bi-dispersed porous media. In order to predict the permeability of bi-dispersed porous media, finite element simulations are performed using COMSOL V3.4 (COMSOL Inc.). COMSOL is known to produce an accurate solution when a simple geometry is considered. In the numerical study, the cluster is modeled as a smooth porous sphere instead of agglomerated particles as shown in figure 8 . The permeability of the sphere is equal to the permeability of mono-dispersed medium as given in equation 1 and thus, is a function of particle diameter. Detailed numerical schemes of figure 8 are identical with those of figure 6 . From a comprehensive numerical study, a correlation for permeability of bi-dispersed medium is developed by fitting the results from a wide range of numerical simulations on various geometries. The result is obtained as the following:

$$
K_{\mathrm{bi}}=\frac{\varepsilon^{3} D_{c}^{2}}{150(1-\varepsilon)^{2}}\left[1+4.03 \exp \left(3.29 \frac{D_{p}}{D_{c}}\right)\left(\frac{D_{p}}{D_{c}}\right)^{2}\right]
$$

In equation 10 , the $\varepsilon$ indicates the porosity of the monodispersed porous media. Equation 10 shows an excellent agreement with the numerical result within the error of $8 \%$ when $0.003<D_{\mathrm{p}} / D_{\mathrm{c}}<0.3$. The first term in equation 10 is the Blake-Kozeny equation and represents the permeability through the large pores. The second term is a correction term for accounting the permeability through the small pores.
In regard to the bi-dispersed porous media, the effect of the cluster size distribution is considered using the same analytic method for the mono-dispersed porous media, which is represented by equations 3-5. Figure 9 shows the results. As shown in this figure, the nondimensional permeability of the bi-dispersed porous media quasilinearly decreases with increasing non-dimensional cluster size distribution range. However, the permeability of the bi-dispersed porous media is not shown to significantly depend on the non-dimensional particle size distribution range. It is because the permeability of the bidispersed porous media is mainly governed by the large pores developed between clusters. In other words, the first term of equation 10 is generally much larger than the second term of the equation.

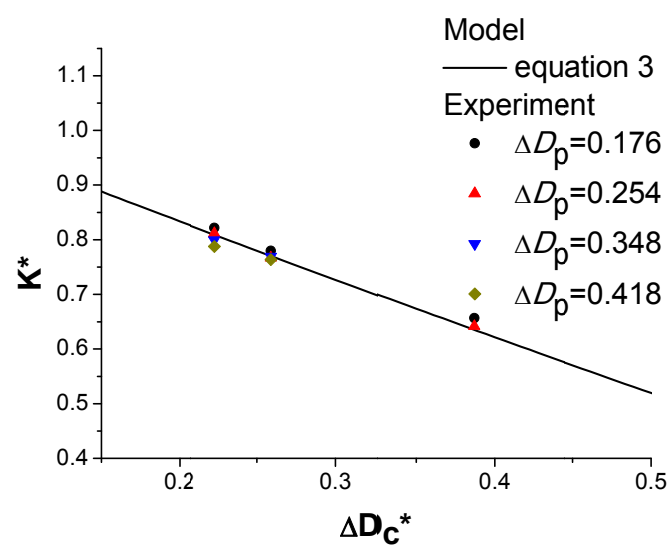

Fig. 9 Comparative plots of analytically and numerically obtained permeabilities versus cluster size distribution

\section{Conclusion}

In this study, the permeability' of mono- and bi-dispersed porous media is considered. The effects of the particle size distribution and the packing structure of particles on the permeability are investigated experimentally and analytically. Both experimental and analytic results suggest that the particle size distribution is close to the log-normal distribution, and the permeability of the mono-dispersed porous media quasi-linearly decreases as the range of the particle size distribution increases. On the other hand, the effect of packing structure of particles on the permeability is shown to be negligible. The permeability of the bi-dispersed porous media quasilinearly decreases as the range of cluster size increases, and nearly independent of the particle size distribution. The present model is valid over the range of parameters typically found in heat transfer applications

\section{Acknowledgement}

This work was supported by the National Research Foundation of Korea (NRF) grant funded by the Korea government (MEST) (No. 2012R1A3A2026427). 


\section{References}

1. A. Faghri, Heat pipe science and technology (Taylor \& Francis, Washington, 1995)

2. C.B. Sobhan, R.L. Rag, G.P. Peterson, Int. J. Energ. Res. 31, 664-688 (2007)

3. C. Byon, S.J. Kim, Int. J. Heat Mass Transfer 55, 7884-7891(2012)

4. G.P. Peterson, An Introduction to heat pipes (John Wiley \& Sons, Inc., New York, 1998)

5. J.A. Weibel, S.V. Garimella, M.T. North, Int. J. Heat Mass Transfer 53 4204-4215 (2010)

6. M. Kaviany, Principles of heat transfer in porous media (Springer, New York, 1995)

7. T. Semenic, Y.Y. Lin, I. Catton, J. Heat Transfer 130 022602 (2008)

8. C. Byon, Study on the thermal performance of bioinspired wicks for heat pipe (Ph.D. Dissertation, KAIST, Korea, 2012)

9. C. Byon, S.J. Kim, Int. J. Heat Mass Transfer 55 4096-4103 (2012)

10. C. Byon, S.J. Kim, J. Micromech. Microeng. 21 $115011(2011)$

11. Y.S. Choi, S.J. Kim, D. Kim, Transport Porous Med. 75 133-149 (2008)

12. M.J. MacDonald, C.F. Chu, P.P. Guilloit, K.M. Ng, AIChE J. 37 1583-1588 (1991)

13. J. Heintzenberg, Aerosol Sci. Tech. 21 46-28 (1994)

14. E.L. Crow, K. Shimizu, Log-normal distributions: theory and application (New York: Dekker, 1988)

15. F.P. Incropera, D.P. DeWitt, Fundamentals of heat and mass transfer (John Wiley \& Sons, Inc., New York, 2002) 